\title{
Optimization of Human Resource Management
}

\author{
Musheng Tian
}

School of Mathematics and Physics, China University of Geosciences, P.R. China

*Corresponding author: Musheng Tian, Associate Professor, tms64@126.com.

\begin{abstract}
Whether government agencies, enterprises or institutions, in the implementation of job settings, are related to the rational allocation of human resources. In this paper, the human resource management should be followed two principles: to make the best of their talents and set up posts on demand, its essence is the economic efficiency and work efficiency. According to these two principles, the use of integer programming and dynamic programming, the establishment of appropriate mathematical model, the system indicators are optimized to improve the economic efficiency of units and work efficiency. The advantages and disadvantages of each model are briefly analyzed. The conclusion provides a certain theoretical basis for the post setting of each unit.
\end{abstract}

Key words: human resources; post settings; economic benefits; working efficiency; integer programming; dynamic programming

\section{Introduction}

At present, in order to maximize the benefits, all units at all levels are the widespread implementation of post appointment system, as far as possible to give full scope to the talents and according to the need to set up post. There are many literatures about the research position. Position set up an important principle is based on the nature of the unit and the actual need to set a number of departments, and then according to the workload of the departments set up jobs, that is, "according to the need to set up post".

For ease of discussion, to a group company, for example, the company based on the number of branches, number of branches and departments tasks of various departments, and then determine the demand for all kinds of talents according to the standard workload of various types of employees, which is required all kinds of several post settings. The ideal state is the number of the number of such positions is exactly equal to the existing types of employees should be set, but less likely to occur in this state. The general state of the number of various types of existing employees more or less than the number of such positions should be set for this situation also has a variety of solutions, such as recruitment, transfer of the contract, dismissal, etc., are not discussed in detail here. This paper considers the various branches of the same department as a system, in this system, the overall optimization of existing staff placement, in order to achieve optimal overall economic efficiency and productivity. This paper tries to use integer programming and dynamic programming to solve this problem.

\section{Establish the principle of mathematical model}

Mathematical model of the system set up staff positions should follow the following two principles: 


\subsection{Economic Benefits}

If the workload of the staff to exceed its standard workload, you need employees to work overtime, the company is required to pay overtime; if the employee's work amounted to less than their standard workload, the staff time with nothing to do, will result in waste of human resources . Starting from the interests of staff overtime should be less, from the interests of the company nothing to do starting time should be less. In order to maximize economic efficiency, the total amount of employees' overtime hours and nothing to do time should be as small as possible.

\subsection{Working efficiency}

Each employee's time and energy is limited, too much overtime affect work quality and efficiency. From this point of view should also minimize overtime. In addition, employees want to work in a more balanced system, but the efficiency of each employee are not the same, so the same amount of work each employee is unrealistic. Workload among employees that much difference will affect the efficiency of the entire system.

\section{Establishing mathematical model}

Suppose there are $m$ Group subsidiaries, a total of $N$ sector employees in total working week $k$-branch of the department of $A_{k}$, each employee week standard working time of 40 hours. According to the above two principles, we can establish the following three mathematical models:

\subsection{Total overtime minimization model}

Let $x_{k}$ denote the number of employees in the sector $k$-branch set, $S_{k}\left(x_{k}\right)$ represents the total overtime $k$-branch of the sector, then

$$
S_{k}\left(x_{k}\right)=A_{k}-40 x_{k}
$$

Then there are the model ( I )

$$
\begin{gathered}
\min \sum_{k=1}^{m} S_{k}\left(x_{k}\right) \\
\begin{cases}\sum_{k=1}^{m} x_{k}=N \\
S_{k}\left(x_{k}\right) \geq 0 & (k=1,2, \cdots, m) \\
x_{k} \in Z^{+} & (k=1,2, \cdots, m)\end{cases}
\end{gathered}
$$

In case of a shortage of staff, the optimal solution can be obtained ( I ), so that the total overtime entire company in the sector to a minimum. But the per capita overtime This solution may cause the system in all branches of the imbalance, that is to say some more overtime hours per branch, per capita less overtime and some branches, and therefore need to improve the model.

\subsection{Overtime the most balanced model}

By $T_{k}\left(x_{k}\right)$ represents the $k$ branch of the sector average overtime hours, that 


$$
T_{k}\left(x_{k}\right)=\frac{A_{k}}{x_{k}}-40
$$

Ceiling $t$ to control overtime per capita, an increase of constraints $T_{k}\left(x_{k}\right) \leq t$, then there is the model ( II):

$$
\begin{gathered}
\min \sum_{k=1}^{m} S_{k}\left(x_{k}\right) \\
\begin{cases}\sum_{k=1}^{m} x_{k}=N \\
S_{k}\left(x_{k}\right) \geq 0 & (k=1,2, \cdots, m) \\
T_{k}\left(x_{k}\right) \leq t & (k=1,2, \cdots, m) \\
x_{k} \in Z^{+} & (k=1,2, \cdots, m)\end{cases}
\end{gathered}
$$

When the model ( II ) of non-empty feasible region optimal solutions exist when the feasible region is the empty set may be appropriate to increase the value of $t$, so that the feasible region is not empty. To get the lowest per capita overtime, you can gradually decrease the value of $t$ until a non-empty feasible region to meet the minimum value appears $t$, the maximum limit at this optimal solution corresponding to the minimum per capita overtime hours, overtime will reach the most balancing purposes. With the addition of constraint $T_{k}\left(x_{k}\right) \leq t$, narrowing the feasible region, the model ( II ) total overtime optimal solution may be greater than the corresponding model ( I ).

\subsection{Total time per overtime period and per capita nothing to do minimal model}

If you remove the limit $S_{k}\left(x_{k}\right) \geq 0$, then when $S_{k}\left(x_{k}\right)<0, S_{k}\left(x_{k}\right)$ represents the $k$ branch of the division's total nothing to do time, $T_{k}\left(x_{k}\right)$ represents the $k$ branch of the sector per capita nothing to do time. So $\min \sum_{k=1}^{m}\left|T_{k}\left(x_{k}\right)\right|$ represents the sum of overtime per system and per capita nothing to do and time is minimized. Since $T_{k}\left(x_{k}\right)$ is nonlinear, and discrete and therefore solved by dynamic programming.

When determining each branch of the sector number of jobs as a stage multi-stage decisionmaking process, provided in the first $1,2, \ldots, i$ branch, the department set up a total of $N_{i}$ jobs per capita overtime and all branches per capita nothing to do time minimum sum of $f_{i}\left(N_{i}\right)$, according to the optimization principle, the dynamic programming model (

III)

$$
\left\{\begin{array}{c}
f_{i}\left(N_{i}\right)=\min _{x_{i}}\left[\left|T_{k}\left(x_{k}\right)\right|+f_{i-1}\left(N_{i}-x_{i}\right)\right] \quad(i=2,3, \cdots, m) \\
f_{1}\left(N_{1}\right)=\left|T_{1}\left(x_{1}\right)\right|=\left|T_{1}\left(N_{1}\right)\right|
\end{array}\right.
$$

Where $f_{m}\left(N_{m}\right)=f_{m}(N)$ is the whole system of the sector average overtime hours and the minimum sum per capita nothing to do time, that

$$
\min \sum_{k=1}^{m}\left|T_{k}\left(x_{k}\right)\right|=f_{m}\left(N_{m}\right)=f_{m}(N) .
$$

When calculating $f_{i}\left(N_{i}\right)$, the same request $T_{k}\left(x_{k}\right) \leq t$, to narrow the range of $N_{i}$. 
When calculating $f_{1}\left(N_{1}\right), T_{1}\left(x_{1}\right)=\frac{A_{1}}{x_{1}}-40\left(x_{1}>0\right)$ is a decreasing function of $x_{1}$, let $T_{1}\left(x_{1}^{\prime}\right) \leq t, T_{1}\left(x_{1}^{\prime \prime}\right)<0, x_{1}^{\prime}, x_{1}^{\prime \prime}$ is an integer and the first time, the $N_{1}$ is in the range of $\left[x_{1}^{\prime}, x_{1}^{\prime \prime}\right]$ all integers on. This is because in this range of $x_{1}$ satisfies $T_{1}\left(x_{1}\right) \leq t$, when $x_{1}>x_{1}^{\prime \prime}$, $\left|T_{1}\left(x_{1}\right)\right|>\left|T_{1}\left(x_{1}^{\prime \prime}\right)\right|$, so that the $x_{1}>x_{1}^{\prime \prime}$ range excluded.

For $f_{2}\left(N_{2}\right)$, set $T_{2}\left(x_{2}^{\prime}\right) \leq t, T_{2}\left(x_{2}^{\prime \prime}\right)<0, x_{2}^{\prime}, x_{2}^{\prime \prime}$ is an integer and the first time, the $N_{2}$ is in the range of $\left[x_{2}^{\prime}+N_{1}^{\prime}, x_{2}^{\prime \prime}+N_{1}^{\prime \prime}\right]$ on all integers, $\left[N_{1}^{\prime}, N_{1}^{\prime \prime}\right]$ is in the range of $N_{1}$.

In general, for $f_{i}\left(N_{i}\right)$, set $T_{i}\left(x_{i}^{\prime}\right) \leq t, T_{i}\left(x_{i}^{\prime \prime}\right)<0, x_{i}^{\prime}, x_{i}^{\prime \prime}$ is an integer and the first time, the $N_{i}$ is in the range of $\left[x_{i}^{\prime}+N_{i-1}^{\prime}, x_{i}^{\prime \prime}+N_{i-1}^{\prime \prime}\right]$ on all integers, $\left[N_{i-1}^{\prime}, N_{i-1}^{\prime \prime}\right]$ is in the range of $N_{i-1}$.

\section{Conclusion}

This article research tool to optimize human resources management with integer programming and dynamic programming, which aims to make full use of existing human resources through appropriate allocation, to maximize the economic benefits of the company, the highest efficiency of the work. The three mathematical models have their own strengths, the total overtime Model ( I ) given the smallest, but the workload of all branches of the sector most unequal, that some overtime branch more, some branches without nothing to do more time; workload model ( II ) gives the most balanced, but the total overtime hours may be greater than the model ( I ); overtime per capita model (III) and per capita given nothing to dry The minimum sum of the time, but can not guarantee a balanced workload. This requires managers to be selected according to the actual situation.

\section{References}

1. J.P. Xu, Z.N. Hu, S. Wang, Operations research [M]. 2nd ed. Beijing: Science Press, 2004.

2. Y.Q. Hu, Y.H. Ge, Operational Research Tutorial [M]. 3rd ed. Beijing: Tsinghua University Press .2007.

3. H.L. Ding, F. Wang, Model and Algorithm Research on Human Resource Optimization[J]. Science Technology and Engineering.2009,01.76-79

4. Z.B. Yang, F. Dai, G.H. Nie, Faculty positions in universities and set up model, Journal of Wuhan University of Technology (Social Sciences Edition), 2009,03.107110. 